\title{
Radiation- and Bound-Mode Propagation in Rectangular, Multimode Dielectric, Channel Waveguides With Sidewall Roughness
}

\author{
Ioannis Papakonstantinou, Member, IEEE, Member, OSA, Richard James, and David R. Selviah, Member, IEEE
}

\begin{abstract}
This paper calculates and displays accurate radiation modes for rectangular, multimode dielectric, channel waveguides, for the first time, and introduces the new semianalytical calculation method used to find them, the radiation-mode Fourier decomposition method (RFDM), which is an extension of the Fourier decomposition method (FDM) for finding bound propagating modes. The optimum choice of non-linear conformal transformation parameters is discussed for achieving highest accuracy. Once the radiation modes are known, the coupling coefficients can be found between the bound and radiation modes, as well as those between the bound modes themselves, andhence, the propagation loss can be found. The paper adapts Marcuse's coupled power theory, for the first time, to enable it to model propagation in rectangular, multimode dielectric, channel waveguides suffering from one dimensional sidewall roughness enabling the equilibrium distance to be calculated, at which rate of loss to radiation modes becomes constant, and to find that equilibrium propagation loss, and the dependence on the statistical properties of the wall roughness. This leads to the conclusion that at sufficient distance there exist two uncoupled modes, a symmetric and an asymmetric lowest order mode.
\end{abstract}

Index Terms-Coupled power theory, optical interconnects, propagation loss, radiation modes, sidewall roughness.

\section{INTRODUCTION}

W AVEGUIDE sidewall roughness has been the subject of a number of papers in optics and photonics [1]-[25], since it is one of the main causes of propagation loss due to scattering apart from material absorption, in single-mode and multimode waveguides. Light propagation in the presence of roughness is a particularly challenging problem, especially in multimode waveguides, as energy continuously redistributes itself between the guided modes and radiates from the waveguide core into the cladding due to coupling between bound and radiation modes. Marcuse stated in his coupled mode and coupled power theories [1]-[8] that as modes propagate in a waveguide with randomly deformed sidewalls they converge to a specific

Manuscript received December 16, 2008; revised April 27, 2009. First published May 12, 2009; current version published August 21, 2009. This work was supported by the U.K. Engineering and Physical Sciences Research Council (EPSRC) under the Flagship Project DTA and IeMRC OPCB Flagship. The work of I. Papakonstantinou was supported by Xyratex Technology Ltd.

I. Papakonstantinou was with the Optical Devices and Systems Laboratory, Department of Electronic and Electrical Engineering, University College London (UCL), London WC1E 7JE, U.K. He is now with the CERN-European Organization for Nuclear Research, Geneva 23, Switzerland.

R. James and D. R. Selviah are with the Optical Devices and Systems Laboratory, Department of Electronic and Electrical Engineering, University College London (UCL), London WC1E 7JE, U.K. (e-mail: d.selviah@ee.ucl.ac.uk).

Color versions of one or more of the figures in this paper are available online at http://ieeexplore.ieee.org.

Digital Object Identifier 10.1109/JLT.2009.2022766 distribution, which he called the equilibrium distribution. The average power ratio between modes remains constant at equilibrium, although the instantaneous power ratios might vary, and the power distribution between the modes depends on the statistical properties of the waveguide wall roughness, namely its standard deviation and autocorrelation length. The propagation loss and modal power distribution vary as modes propagate in the transition region before reaching an equilibrium distribution and in that region they depend on the excitation source and its position and orientation. The required distance to reach equilibrium was called the equilibrium length. The idea of an equilibrium distribution is important since at equilibrium the average propagation loss per unit waveguide length becomes a constant independent of the initial field that excited the waveguide.

Until recently, coupled mode theory had not been applied to rectangular cross section waveguides directly. The main reason for this was that analytic solutions for the radiation modes, which play a central role in the theory, did not exist. Researchers tried to deal with this problem by either considering a 2-D effective refractive index approximation of the waveguide [9], [10] or by approximating the radiation modes with the free-space cladding modes [11]-[13]. However, both of these methods are only approximate, and in some cases, might be rather inaccurate. A precise method was presented in [14], where the actual radiation modes of a high-index contrast nanowire were presented. However, the waveguides examined there were single mode, and so the focus was on the propagation loss alone.

Other methods that have dealt with the subject in the past included the volume current method [15]-[18], finite-difference time domain (FDTD) [14], and ray tracing [19]. The volume current method treats roughness as an equivalent current source and loss is calculated by the outgoing radiation in the far field. In raytracing techniques, modes are approximated by plane waves and loss is calculated by the scattering matrices of the plane waves on the randomly perturbed waveguide sidewalls. Although some of these methods have demonstrated good agreement with experiments they are either intended only for single-mode operation (volume current method), or they are too time consuming for multimode waveguides (FDTD) or, finally, they can only provide information about the propagation loss (ray tracing).

This study demonstrates, for the first time, the radiation modes and the e act application of the coupled power theory in rectangular cross-section, highly multimode waveguides. Radiation modes are calculated by e tending the Fourier decomposition method (FDM) for bound modes, which was first presented in [20] and later modified in [21]. We refer to our method as the radiation-mode FDM (RFDM) in the following 


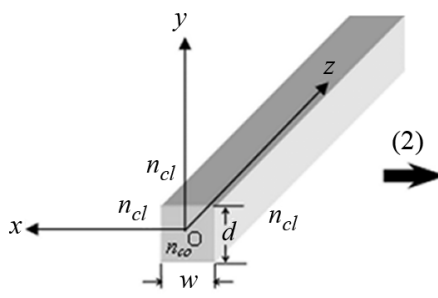

(a)

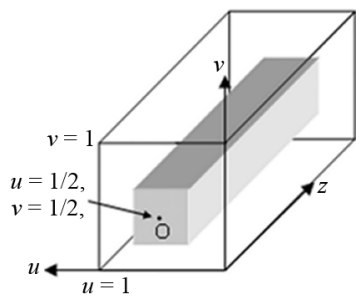

(b)
Fig. 1. (a) Rectangular waveguide in a Cartesian coordinate system with width $w$ and thickness $d$. Cladding refractive index is $n_{\mathrm{cl}}$ while core refractive index is $n_{\mathrm{co}} \cong n_{\mathrm{cl}}$. (b) Same waveguide in the transformed $u v$-domain.

to distinguish it from FDM. Upon calculation of the radiation modes, the coupled power theory is applied to a $50 \mu \mathrm{m} \times 50 \mu \mathrm{m}$ test multimode waveguide and the equilibrium loss, length and power distribution as a function of the standard deviation, and autocorrelation length of roughness are calculated.

This paper is arranged as following. Section II describes the RFDM algorithm and its differences from FDM. Section III validates the RFDM by applying it to a slab waveguide and by comparing RFDM results with existing analytic solutions. Section IV extends the coupled power theory to rectangular dielectric waveguides. Section V presents the results for equilibrium propagation loss, equilibrium length, and equilibrium power distribution for a series of test waveguides typically used in optical interconnects, and finally, Section VI presents the conclusion.

\section{RADIATION-MODE Fourier DECOMPOSITION METHOD}

A rectangular waveguide, such as the one shown in Fig. 1(a), can support two types of modes differentiated by their polarization. Modes whose dominant electric field component is mainly polarized along $x$, are called $E_{x}$ or $T E$-type, while modes whose electric field is polarized along $y$, are called $E_{y}$ or TM-type [26]. In the weakly waveguiding limit where the cladding index is similar to the core index, the two polarizations become almost indistinguishable and, therefore, in the following, we focus on the $E_{x}$ set of modes, although $E_{y}$ modes could be considered as well if necessary. Bound modes have propagation constants $\beta_{b}$, limited in the region $k n_{\mathrm{cl}}<\beta_{b}<n_{\mathrm{co}}$, while propagation constants of the radiation modes $\beta_{r}$, lie in the region $0<\beta_{r}<$ $k n_{\text {cl }}$, for forward propagation, where $k=2 \pi / \lambda$ denotes the free-space wavenumber, and $\lambda$ the free-space wavelength.

The dominant transverse electric field component satisfies the 3-D scalar wave equation everywhere in space, which for a mode with propagation constant $\beta$ (bound or radiation), takes the usual form (time dependence omitted) in a Cartesian coordinate system [26]

$$
\left(\frac{\partial^{2}}{\partial x^{2}}+\frac{\partial^{2}}{\partial y^{2}}+k^{2} n^{2}(x, y)-\beta^{2}\right) E_{x}(x, y)=0
$$

The refractive index distribution $n(x, y)$ [see Fig. 1(a)] equals to $n_{\mathrm{co}}$ inside the core and $n_{\mathrm{cl}}$ elsewhere. Bound mode FDM relies on the nonlinear conformal transformation (2), in which the infinite $x y$-plane is mapped onto a unit square in an alternative $u v$-coordinate system shown in Fig. 1(b).

$$
x=p \tan \left[\pi\left(u-\frac{1}{2}\right)\right], \quad y=q \tan \left[\pi\left(v-\frac{1}{2}\right)\right] \text {. }
$$

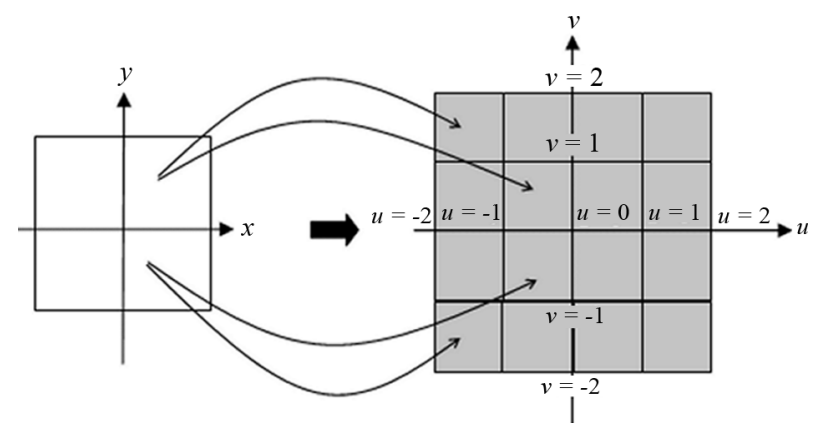

Fig. 2. Periodic property of transformation (2). Original $x y$-domain is mapped into an infinite number of periodically arranged unit squares in the $u v$-domain, with period of 1 unit length in both $u$ - and $v$-directions.

As described in [21], $p$ and $q$ in (2) are free scaling parameters, which if chosen appropriately, yield rapid convergence. Upon substitution of (2) into (1), the transformed wave equation in the $u v$-coordinate system becomes

$$
\begin{aligned}
\left\{\left(\frac{\partial u}{\partial x}\right)^{2} \frac{\partial^{2}}{\partial u^{2}}+\frac{\partial^{2} u}{\partial x^{2}} \frac{\partial}{\partial u}+\left(\frac{\partial v}{\partial y}\right)^{2} \frac{\partial^{2}}{\partial v^{2}}\right. & \\
+ & \left.\frac{\partial^{2} v}{\partial y^{2}} \frac{\partial}{\partial v}+k^{2} n^{2}(u, v)-\beta^{2}\right\} E_{x}(u, v)=0 .
\end{aligned}
$$

Bound modes, must satisfy the Sommerfeld radiation condition, stating that fields as well as their transverse derivatives, should tend to zero as $x \rightarrow \pm \infty$ or $y \rightarrow \pm \infty$, which, in turn, translates to a zero field boundary condition when $u \rightarrow 0,1$ or $v \rightarrow 0,1$ in the transformed domain. The authors in [21] used this property to apply equivalent metallic boundary conditions at $u=0,1$ and $v=0,1$ and solutions for $E_{x}$, were expressed as a linear superposition of a set of sinusoidal basis functions, (4a), with zero values at the boundaries of the square

$$
E_{z}(u, v)=4 \sum_{m=1}^{M} \sum_{n=1}^{N} c_{m n} \sin (m \pi u) \sin (n \pi v),
$$

where $c_{m n}$ are the coefficients of the double Fourier series in (4a).

At this point RFDM starts to depart from FDM. Two crucial observations are e ploited in the calculation of radiation modes. First, transformation (2) is not unique but in fact a periodic mapping of the $x y$-space into the $u v$-space as shown in Fig. 2. This feature serves as the basis to define the Fourier series for the radiation modes. Second, neither the Sommerfeld nor Neumann boundary conditions are strictly satisfied by radiation modes and, therefore, solutions should be allowed to take arbitrary values at the boundaries of each unit square. Based on these observations, radiation modes may be represented by the following 2-D Fourier series:

$$
\begin{aligned}
E_{x}^{r}(u, v)= & 4 \sum_{l=0}^{+\infty} \sum_{m=0}^{+\infty}\left\{C_{l m}^{r, \mathrm{oo}} S_{l m}^{\mathrm{oo}}(u, v)\right. \\
& +C_{l m}^{r, \mathrm{eo}} S_{l m}^{\mathrm{eo}}(u, v)+C_{l m}^{r, \mathrm{oe}} S_{l m}^{\mathrm{oe}}(u, v) \\
& \left.+C_{l m}^{r, \mathrm{ee}} S_{l m}^{\mathrm{ee}}(u, v)\right\} \\
= & E_{x}^{r, \mathrm{oo}}(u, v)+E_{x}^{r, \mathrm{eo}}(u, v) \\
& +E_{x}^{r, \mathrm{oe}}(u, v)+E_{x}^{r, \mathrm{ee}}(u, v)
\end{aligned}
$$


with the following abbreviations for the basis functions: $S_{l m}^{\text {oo }}(u, v)=\sin (2 l \pi u) \sin (2 m \pi v), S_{l m}^{\mathrm{eo}}(u, v)=$ $\cos (2 l \pi u) \sin (2 m \pi v), S_{l m}^{\mathrm{oe}}(u, v)=\sin (2 l \pi u) \cos (2 m \pi v)$, $S_{l m}^{\mathrm{ee}}(u, v)=\cos (2 l \pi u) \cos (2 m \pi v)$, and $C_{l m}^{r, \mathrm{oo}}, C_{l m}^{r, \mathrm{eo}}, C_{l m}^{r, \mathrm{oe}}$, and $C_{l m}^{r, \text { ee }}$ the amplitude coefficients of each one of the four double series, respectively. Equation (4b) consists of a combination of orthonormal cosinusoidal and sinusoidal functions with a fundamental spatial period of 1 unit length in the $u$-direction and 1 unit length in the $v$-direction to embody the periodicity of the transformation (2). Sinusoidal terms express fields with odd symmetry about planes $u=(1 / 2+k)$ or about planes $v=(1 / 2+k), k=0, \pm 1, \pm 2, \pm 3, \ldots$, while cosinusoidal terms express fields with even symmetry. The way these sinusoidal and cosinusoidal basis functions enter (4b) implies that the total field is expressed as a linear combination of four fields, each one possessing a different symmetry combination in the $u$-direction (or equivalently in the $x$-direction) and in the $v$-direction (or equivalently in the $y$-direction). According to the order they appear in (4b), these symmetries are the odd-odd $\left(E_{x}^{r, \text { oo }}\right)$, the even-odd $\left(E_{x}^{r, \text { eo }}\right)$, the odd-even $\left(E_{x}^{r, \text { oe }}\right)$, and the even-even $\left(E_{x}^{r, e e}\right)$, respectively.

To calculate the amplitude coefficients for a given radiation mode in (4b), we assume that this radiation mode consists of a known free-space part, and a response field due to the presence of the waveguide. This in effect means that we treat each radiation mode as a perturbation of a free-space propagating wave, as explained in [14] and [27]. By applying this perturbation method, we gain not only the knowledge of the yet undetermined amplitude coefficients $C_{l m}^{i j}$, but in addition, a means to normalize the radiation modes. The earlier steps are another significant departure from FDM . There, the amplitude coefficients of the bound modes were calculated by substituting the Fourier series (4a) into the transformed wave (3), multiplying by a basis harmonic function, and integrating over the unit square. This process led to an eigenvalue equation whose solutions corresponded to the discrete set of propagation constants of the bound modes.

However, if the same approach was followed by RFDM, the determinant of the system for the radiation modes would be nonvanishing so that the propagation constants form a continuum [2, p. 23]. Such an equation would not be soluble for any radiation mode propagation constant $\beta_{r}$, and so it would not give the amplitude coefficients of the Fourier series.
To calculate the amplitude coefficients for the radiation mode Fourier series, we proceed as following. Equation (3) is recast in the convenient operator form

$$
\left(L_{f}+k^{2} N^{2}(u, v)-\left(\beta^{2}-k^{2} n_{\mathrm{cl}}^{2}\right)\right) E_{x}^{r}(u, v)=0
$$

where the $L_{f}$ operator in (5) is defined as

$$
\begin{aligned}
L_{f}=\left(\frac{\partial u}{\partial x}\right)^{2} \frac{\partial^{2}}{\partial u^{2}} & +\frac{\partial^{2} u}{\partial x^{2}} \frac{\partial}{\partial u} \\
& +\left(\frac{\partial v}{\partial y}\right)^{2} \frac{\partial^{2}}{\partial v^{2}}+\frac{\partial^{2} v}{\partial y^{2}} \frac{\partial}{\partial v}+k^{2} n_{\mathrm{cl}}^{2}
\end{aligned}
$$

and

$$
N^{2}(u, v)= \begin{cases}0, & \text { in the waveguide cladding } \\ n_{\mathrm{co}}^{2}-n_{\mathrm{cl}}^{2}, & \text { in the waveguide core. }\end{cases}
$$

Then, the radiation-mode field $E_{x}^{r}(u, v)$ is expressed as the combination of a free-space part $E_{x}^{f}(u, v)$ and a response part $E_{x}^{R}(u, v)$ as

$$
E_{x}^{r}(u, v)=E_{x}^{f}(u, v)+E_{x}^{R}(u, v)
$$

and (8) is substituted into (5) to obtain

$$
\begin{array}{r}
\left(L_{f}+k^{2} N^{2}(u, v)-\left(\beta^{2}-k^{2} n_{\mathrm{cl}}^{2}\right)\right) E_{x}^{R}(u, v) \\
=k^{2} N^{2}(u, v) E_{x}^{f}(u, v) .
\end{array}
$$

Equation (9) is derived using the fact that since $E_{f}^{x}(u, v)$ is a free-space field, it has to satisfy the free-space wave equation

$$
\left(L_{f}-\left(\beta^{2}-k^{2} n_{\mathrm{cl}}^{2}\right)\right) E_{x}^{f}(u, v)=0 .
$$

Equation (4b) is next split into free-space and response components (see (11) at the bottom of the page) and, finally, the expressions for $E_{x}^{f}(u, v)$ and $E_{x}^{R}(u, v)$ are substituted from (11) into (9), and the result is multiplied by a basis function $S_{l^{\prime} m^{\prime}}^{i j}(u, v)(i, j=e$ or $o)$, and integrated over any of the unit squares shown in Fig. 2 to give

$$
\begin{aligned}
\sum_{l=0}^{L} \sum_{m=0}^{M} & \left\{\mathbf{P}_{l m, l^{\prime} m^{\prime}}^{i j}+\mathbf{Q}_{l m, l^{\prime} m^{\prime}}^{i j}-\left(\beta^{2}-k^{2} n_{\mathrm{cl}}^{2}\right)\right. \\
& \left.\times \delta_{l l^{\prime}, m m^{\prime}}\right\} \cdot C_{l m}^{R, i j}=\sum_{l=0}^{L} \sum_{m=0}^{M} \mathbf{P}_{l m, l^{\prime} m^{\prime}}^{i j} \cdot C_{l m}^{f, i j} .
\end{aligned}
$$

$$
\begin{aligned}
E_{x}^{r}(u, v)= & \overbrace{\sum_{l=0}^{+\infty} \sum_{m=0}^{+\infty}\left\{C_{l m}^{f, \mathrm{oo}} S_{l m}^{\mathrm{oo}}(u, v)+C_{l m}^{f, \mathrm{eo}} S_{l m}^{\mathrm{eo}}(u, v)+C_{l m}^{f, \mathrm{oe}} S_{l m}^{\mathrm{oe}}(u, v)+C_{l m}^{f, \mathrm{ee}} S_{l m}^{\mathrm{ee}}(u, v)\right\}}^{E_{x}^{f}(u, v)} \\
& +\overbrace{\sum_{l=0}^{+\infty} \sum_{m=0}^{+\infty}\left\{C_{l m}^{R, \mathrm{oo}} S_{l m}^{\mathrm{oo}}(u, v)+C_{l m}^{R, \mathrm{eo}} S_{l m}^{\mathrm{eo}}(u, v)+C_{l m}^{R, \mathrm{oe}} S_{l m}^{\mathrm{oe}}(u, v)+C_{l m}^{R, \text { ee }} S_{l m}^{\mathrm{ee}}(u, v)\right\}}^{E_{x}^{R}(u, v)}
\end{aligned}
$$


In (12), the upper limits for $l$ and $m$ are truncated from $+\infty$ in (4b), to $L$ and $M$, respectively, which must be large enough to ensure convergence. The matrices in (12) are defined to be

$$
\begin{aligned}
\mathbf{P}_{l m, l^{\prime} m^{\prime}}^{i j}= & \int_{0}^{1} \int_{0}^{1} k^{2} N^{2}(u, v) S_{l m}^{i j}(u, v) \\
& \times S_{l^{\prime} m^{\prime}}^{i j}(u, v) d u d v \\
\mathbf{Q}_{l m, l^{\prime} m^{\prime}}^{i j}= & \mathbf{I}_{1}^{i j}+\mathbf{I}_{2}^{i j}+\mathbf{I}_{3}^{i j}+\mathbf{I}_{4}^{i j} \\
\mathbf{I}_{1}^{i j}= & -l^{2} \pi^{2} \int_{0}^{1} \int_{0}^{1}\left(\frac{\partial u}{\partial x}\right)^{2} \\
& \times S_{l m}^{i j}(u, v) S_{l^{\prime} m^{\prime}}^{i j}(u, v) d u d v \\
\mathbf{I}_{2}^{i j}= & l \pi \int_{0}^{1} \int_{0}^{1}\left(\frac{\partial^{2} u}{\partial x^{2}}\right) \frac{1}{\tan (l \pi u)} \\
& \times S_{l m}^{i j}(u, v) S_{l^{\prime} m^{\prime}}^{i j}(u, v) d u d v \\
\mathbf{I}_{3}^{i j}= & -m^{2} \pi^{2} \int_{0}^{1} \int_{0}^{1}\left(\frac{\partial v}{\partial y}\right)^{2} S_{l m}^{i j}(u, v) \\
& \times S_{l^{\prime} m^{\prime}}^{i j}(u, v) d u d v \\
\mathbf{I}_{4}^{i j}= & m n \pi \int_{0}^{1} \int_{0}^{1}\left(\frac{\partial^{2} v}{\partial y^{2}}\right) \frac{1}{\tan (m \pi v)} \\
& \times S_{l m}^{i j}(u, v) S_{l^{\prime} m^{\prime}}^{i j}(u, v) d u d v .
\end{aligned}
$$

The analytic expressions for (15a)-(15d) are too long to be presented here, and so they are provided in Appendix A.

Equation(12) contains all of the necessary information to calculate the radiation modes. The known free-space field coefficients $C_{l m}^{f, i j}$ provide the unknown response coefficients $C_{l m}^{R, i j}$ by solving the system of linear equations (12). The radiationmode coefficients $C_{l m}^{r, i j}$ then simply follow from (8) and (11) giving

$$
C_{l m}^{r, i j}=C_{l m}^{f, i j}+C_{l m}^{R, i j} .
$$

Once the spectrum coefficients $C_{l m}^{r, i}$ are calculated, the Fourier series ( $4 \mathrm{~b}$ ) is used to calculate the radiation mode in the $u v$-domain. The last step is to use (2) to transform the radiation modes back to the original $x y$-domain. The RFDM algorithm steps are summarized in Fig. 3. An important feature of this method is that only one type of symmetry expressed by the indexes $i$ and $j$, enters (12) at a time. Therefore, radiation modes of different sets of symmetries can be calculated independently, thereby significantly simplifying calculations.

\section{A. Free-Space-Mode Selection}

Radiation modes are to some degree arbitrary since they depend on the choice of the free-space fields used to generate them. A different selection of free-space modes gives rise to a different set of radiation modes. The only requirement for the selected set of free-space modes is that they are complete and orthonormal [27]. Guided by the Cartesian geometry of our system, we choose plane waves to represent the free-space part of the radiation modes. These plane waves are assumed to emerge from four harmonic sources placed at $x=+\infty, x=$

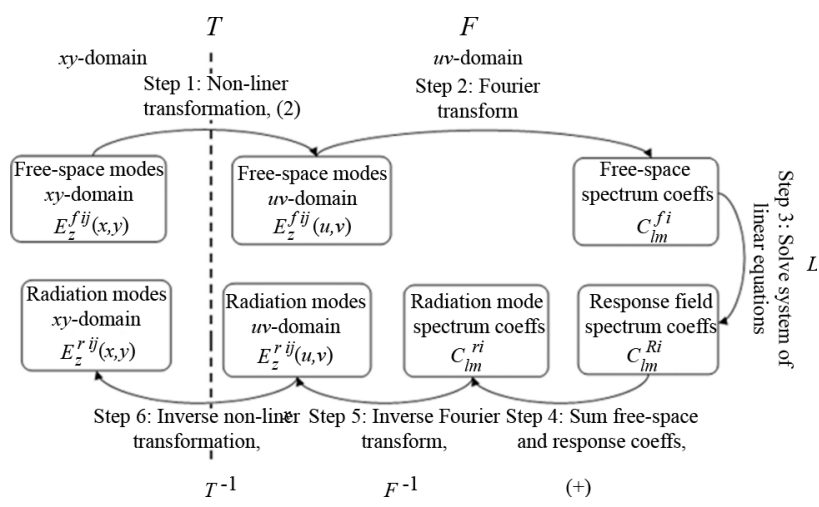

Fig. 3. Algorithm for the calculation of the radiation modes of a rectangular waveguide with the RFDM

$-\infty, y=+\infty$, and $y=-\infty$. The four sources are assumed to oscillate with equal amplitudes and with relative phases determined by the symmetry being considered. The free-space mode with even-even symmetry (even in both $x$ - and $y$-directions), for example, is generated from four plane waves oscillating in-phase as

$$
\begin{aligned}
E_{x}^{f, \mathrm{ee}}(x, y)=\frac{1}{4}( & A^{\mathrm{ee}} e^{+i k_{x} x} e^{+i k_{y} y} \\
& +A^{\mathrm{ee}} e^{-i k_{x} x} e^{+i k_{y} y} \\
& +A^{\mathrm{ee}} e^{+i k_{x} x} e^{-i k_{y} y} \\
& \left.+A^{\mathrm{ee}} e^{-i k_{x} x} e^{-i k_{y} y}\right) \\
=A^{\mathrm{ee}} & \cos \left(k_{x} x\right) \cos \left(k_{y} y\right) .
\end{aligned}
$$

Similarly, for the free-space modes of the other symmetries, we have

$$
\begin{aligned}
& E_{x}^{f, \mathrm{oo}}(x, y)=A^{\mathrm{oo}} \sin \left(k_{x} x\right) \sin \left(k_{y} y\right) \\
& E_{x}^{f, \mathrm{oe}}(x, y)=A^{\mathrm{oe}} \sin \left(k_{x} x\right) \cos \left(k_{y} y\right) \\
& E_{x}^{f, \mathrm{oo}}(x, y)=A^{\mathrm{oo}} \sin \left(k_{x} x\right) \sin \left(k_{y} y\right)
\end{aligned}
$$

where $k_{x}$ is the wavenumber in $x$, and $k_{y}$ the wavenumber in $y$, connected via the usual equation

$$
k_{x}^{2}+k_{y}^{2}+\beta_{r}^{2}=r^{2}+\beta_{r}^{2}=k_{n c l}^{2}
$$

and $r$ the transverse wavenumber $r^{2}=k_{x}^{2}+k_{y}^{2}$. It is now a trivial task to transform the fields in (17a)-(17d), from the $x y$-domain to the $u v$-domain through (2) and Fourier transform the result to calculate the $C_{l m}^{f, i j}$ coefficients for a given $\beta_{r}$ and type of symmetry.

\section{B. Examples of Radiation Modes}

Figs. 4 and 5, show representative selections of radiation modes calculated by RFDM. Fig. 4 shows the odd-odd free-space mode, the response field, and the odd-odd radiation mode for a $50 \mu \mathrm{m} \times 50 \mu \mathrm{m}$ waveguide with $n_{\mathrm{co}}=1.556$, $n_{\mathrm{cl}}=1.5249, \lambda=850 \mathrm{~nm}$, for low spatial frequencies $k=k_{y}=0.1541 \mu \mathrm{m}^{-1}$. The chosen waveguide parameters are typical for optical interconnect applications [28]. Fig. 5(a) and (b) shows the even-even and odd-even free-space and radiation modes, respectively, for the same parameters, as in the previous example. Fig. 5(c) shows an even-even mode, which is of high frequency in the $x$-direction and of low frequency 

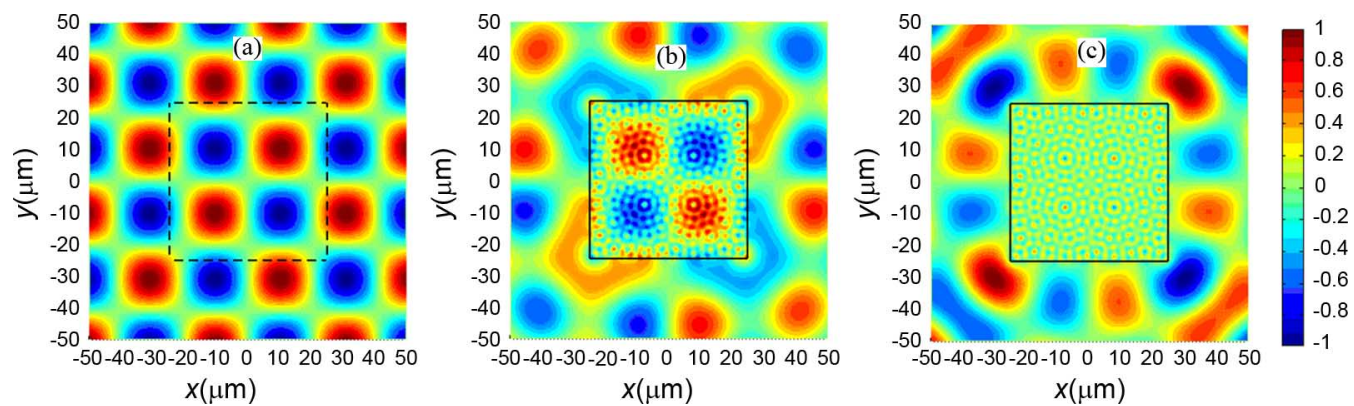

Fig. 4. (a) Free-space mode, (b) response field, and (c) resulting radiation mode for an odd-odd radiation mode with $k_{x}=k_{y}=0.1514 \mu \mathrm{m}^{-1}$. Waveguide parameters: $w=d=50 \mu \mathrm{m}, n_{\mathrm{co}}=1.556, n_{\mathrm{cl}}=1.5249$, and $\lambda=850 \mathrm{~nm}$. Fields have been normalized to unit amplitude for illustration purposes.
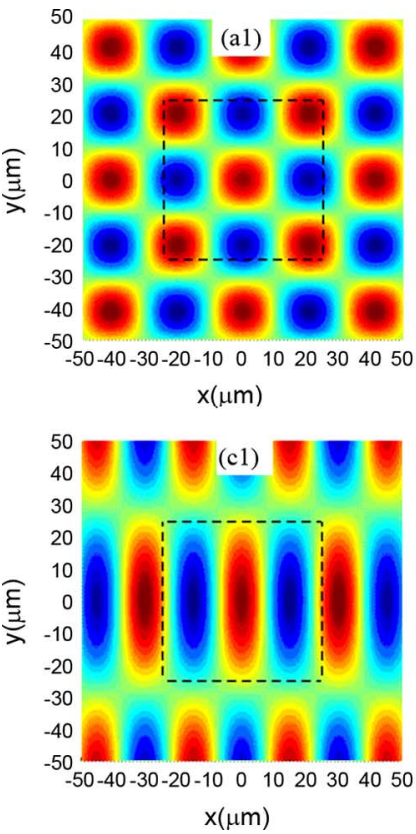
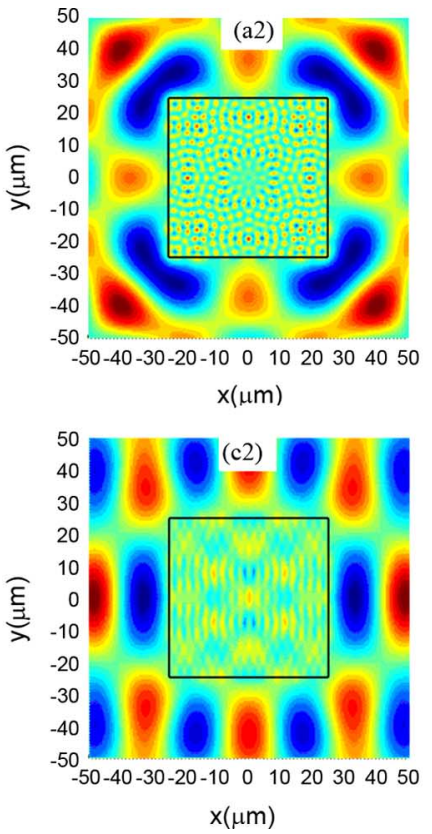
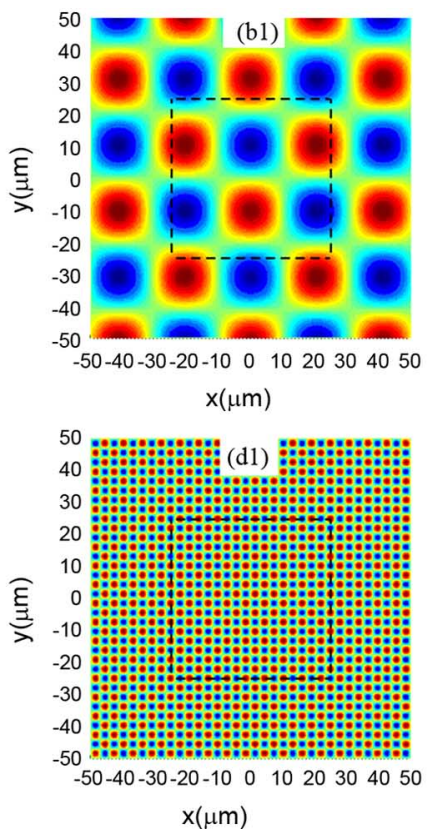
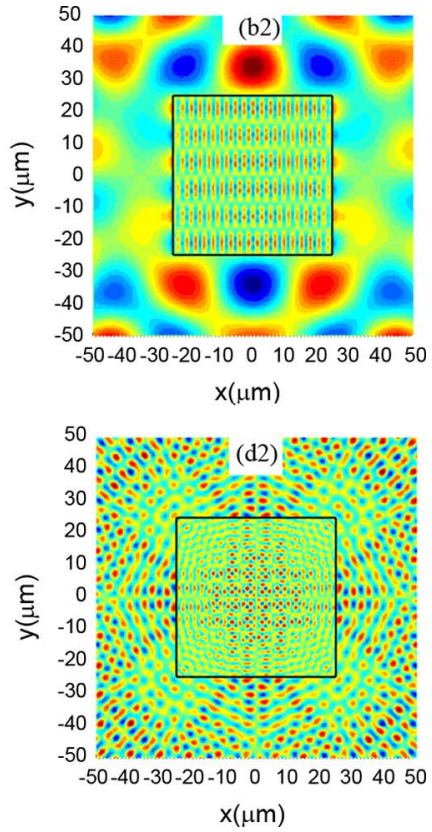

Fig. 5. (a1) and (a2) Free-space mode, and radiation mode for an even-even mode with $k_{x}=k_{y}=0.1514 \mu \mathrm{m}^{-1}$. (b1) and (b2) Free-space mode, and radiation mode for an even-odd mode with $k_{x}=k_{y}=0.1514 \mu \mathrm{m}^{-1}$. (c1) and (c2) Free-space mode, and radiation mode for an even-even mode with $k_{x}=$ $0.2069 \mu \mathrm{m}^{-1}, k_{y}=0.0554 \mu \mathrm{m}^{-1}$. (d1) and (d2) Free-space mode, and radiation mode for an odd-odd mode with $k_{x}=k_{y}=0.8744 \mu \mathrm{m}-1$. Fields have been normalized to unit amplitudes for illustration.

in the $y$-direction $\left(k_{x}=0.2069 \mu \mathrm{m}^{-1}, k_{y}=0.0554 \mu \mathrm{m}^{-1}\right)$, and, finally, Fig. 5(d) shows an odd-odd radiation mode, which is of high spatial frequency in both $x$-and $y$-directions $\left(k_{x}=k_{y}=0.8744 \mu \mathrm{m}^{-1}\right)$. As can be seen in Figs. 4 and 5, radiation modes differ significantly from the free-space modes that generated them and, therefore, it is not advisable for the former modes to be replaced by the latter in propagation loss or other calculations, as has been suggested in [11].

Strictly speaking, the Fourier transforms of the free-space fields in (17a)-(17d), and so of the radiation modes, do not exist everywhere in the $u v$-domain. For example, the even-even free-space mode $E_{x}^{f, \text { ee }}(x, y)$ in (17a), takes the following form in the $u v$-domain:

$$
\begin{aligned}
E_{x}^{f, \mathrm{ee}}(x(u), y(v))= & A^{\mathrm{oo}} \cos \left(k_{x} x\right) \cos \left(k_{y} y\right) \\
= & A^{\mathrm{oo}} \cos \left(p k_{x} \tan \left[\pi\left(\frac{u-1}{2}\right)\right]\right) \\
& \times \cos \left(q k_{x} \tan \left[\pi\left(\frac{v-1}{2}\right)\right]\right) .
\end{aligned}
$$

Due to the tangent functions, this signal has a chirped frequency in the $u$ - and $v$-directions, which steadily increases as we move from the center, $(u, v)=(1 / 2,1 / 2)$, of the unit square toward its edges, $u, v=0$ or $u, v=1$, until it becomes infinite on the boundary. Since an infinite number of oscillations occur within a finite interval, the signals (17a)-(17d) cannot have a Fourier representation close to the boundaries of the unit square. However, their Fourier transform is still valid everywhere else. This minor complication is not restrictive for our purposes since the radiation mode representation fails only at infinity in the original $x y$-domain. Moreover, the application of the coupled mode theory requires the knowledge of the fields very close to the waveguide only, with the information about the fields everywhere else being redundant. Finally, we give a brief e planation about the physical arguments that force the frequency of the free-space mode oscillations to go to infinity. If the frequency of $E_{x}^{f, e e}(u, v)$ were to be finite at $u, v=0$ and $u, v=1$ then the free-space modes (17a)-(17d) would take a well defined value there, which, in turn, would translate to a well defined value at $x= \pm \infty$ and $y= \pm \infty$ in the original $x y$-domain. However, 
this is not possible, as all harmonic functions in (17a)-(17d) are undefined, although bounded, at infinity. Therefore, an infinite frequency at the boundaries of the unit square in the $u v$-domain restores the uncertainty in the harmonic function values at infinity in the $x y$-domain.

\section{VERIFICATION OF RFDM}

In this section, we compare the radiation modes of a slab waveguide calculated by the RFDM with existing analytic expressions for slab radiation modes in order to validate our method. The example of the slab waveguide was selected because it is a Cartesian waveguide geometry where analytic expressions for radiation modes exist.

The core of the slab waveguide under examination extends across the region $-w / 2<x<w / 2$. In the following, we concentrate on the $T E$ radiation modes of the slab waveguide, which have the field components $E_{x}, H_{x}$, and $H_{z}$. According to [2, pp. 21-28], the analytic expressions for the electric field of the even radiation modes, for example, are shown in (20) at the bottom of the page, where the propagation constant $\beta_{r}$ is related to the wavenumber $k_{x}$ inside the core by $k_{x}^{2}+\beta_{r}^{2}=$ $k^{2} n_{\mathrm{co}}^{2}$, and to the wavenumber $\eta$ in the cladding by $\eta^{2}+\beta_{r}^{2}=$ $k^{2} n_{\mathrm{cl}}^{2}$. The radiation modes in (20) have been normalized to unit impulse power flow (power flow, which is a delta function with unit amplitude), as explained in [2].

\section{A. Comparison Between RFDM Calculated and Analytic Slab Radiation Modes}

We now proceed to compare the analytic solutions [see (20)] for the radiation modes to those calculated with the RFDM method for two representative examples. In the course of the comparison, the importance of the scaling parameters $p$ and $q$ in (2) will be demonstrated. Equations(12)-(16) are used for the RFDM calculated radiation modes but with $\partial / \partial y=\partial / \partial u=0$ to reflect the single dimensionality of the problem.

In a first example, a slab waveguide is investigated with the following properties: $w=50 \mu \mathrm{m}, n_{\mathrm{co}}=1.556, n_{\mathrm{cl}}=1.5249$, and $\lambda=850 \mathrm{~nm}$. In this case, the radiation-mode propagation constants are found in the region $0<\beta_{r}<11.272 \mu \mathrm{m}^{-1}$, for forward propagating modes. An even radiation mode with $\beta_{r}=11.20 \mu \mathrm{m}^{-1}$ is examined in order to illustrate the effect of choosing a "very small" value of $p$. Fig. 6(a) plots the radiation mode calculated from the analytic expressions [see (20)], the RFDM calculated mode when $p=3$ and $M=256$, and the difference between the two, in the $u$-domain. On a second $y$-axis, the Cartesian coordinate $x$ as a function of $u$ is plotted. Fig. 6(a) reveals that a "very small" value of $p$ brings the boundaries of the waveguide very close to the boundaries of the unit square in the $u$-domain. In this regime, the waveguide lies in the nonlinear region of the tan function relating $x$ and $u$ via (2), where small deviations in $u$ cause very large deviations in $x$. As a result, although the peaks of the oscillations of the radiation mode are well separated from each other close to $u=1 / 2$, they are brought very close to each other in the vicinity of $u=0$ and $u=1$ resulting in a high-frequency signal. Due to this high spatial frequency, the RFDM algorithm fails to resolve the radiation mode correctly close to the waveguide boundaries for the specific number of Fourier components $M$.

However, the situation changes if a larger value of $p$ is chosen, even though $M$ is kept unchanged. Fig. 6(b) compares the analytic and RFDM radiation mode for $p=50$ this time, again with $M=256$. As shown in Fig. 6(b), the waveguide is now placed within the linear region of the tan function, and both analytic and RFDM radiation modes agree well, at least close to the waveguide boundaries, without having to increase the number of Fourier components, $M$.

On the other hand, allowing $p$ to become arbitrarily large also causes inaccuracies. This is explained with the aid of the second example shown in Fig. 6(c) and (d). In these graphs, the radiation mode with $\beta_{r}=11.20 \mu \mathrm{m}^{-1}$ of a similar slab waveguide is investigated but with $w=20 \mu \mathrm{m}$. Fig. 6(c) compares the analytic with the RFDM calculated mode when $p=200$ and $M=256$. Although the waveguide is clearly within the linear region of the tan function the RFDM algorithm fails again, as can be seen in Fig. 6(c). The explanation is that each radiation mode has a certain number of oscillatory circles inside the waveguide core. A "large" value of $p$ causes the waveguide boundaries to be brought very close to each other in the $u$-domain and, therefore, these oscillations are squeezed within the very narrow waveguide width. This causes a high-frequency signal in the waveguide core this time (rather than close to the unit square boundaries, as in the previous example), which cannot be resolved with the selected value of $M$. Reducing $p$ (but always staying in the linear region of the transformation), gives good agreement between the RFDM algorithm and the analytic solutions, as shown in Fig. 6(d) for the same $M$, but for $p=50$. The two previous examples show that a good choice for $p$ is the one, which balances the frequency components required to accurately reconstruct the radiation mode inside the waveguide and just outside of it. The conclusions from these two examples are summarized in Fig. 7, where we have plotted the normalized rms error between the RFDM and the analytic solution inside the core, as a function of the scaling parameter $p$ for our representative radiation mode with $\beta_{r}=11.20 \mu \mathrm{m}^{-1}, w=50 \mu \mathrm{m}$, and $M=256$. As we see in Fig. 7, for small values of $p$, the error is large, then as $p$ increases it reaches an optimum region beyond which rms error starts increasing again for larger $p$.

Finally, the importance of the number of frequency components $M$, required for convergence is discussed. Different radiation modes require a different number of Fourier components

$$
E_{x}^{e}= \begin{cases}\frac{2 \omega \mu_{o}}{\pi \beta_{r}} \cos \left[\eta\left(x-\frac{w}{2}\right)+a \tan \left(\frac{k_{x}}{\eta} \tan \left(\frac{k_{x} w}{2}\right)\right)\right], & \frac{w}{2}<x<+\infty \\ \frac{2 \omega \mu_{o}}{\pi \beta_{r}}\left[\cos ^{2}\left(\frac{k_{x} w}{2}\right)+\frac{k_{x}^{2}}{\eta^{2}} \sin ^{2}\left(\frac{k_{x} w}{2}\right)\right] \cos \left(k_{x} x\right), & -\frac{w}{2}<x<\frac{w}{2} \\ \frac{2 \omega \mu_{o}}{\pi \beta_{r}} \cos \left[\eta\left(x+\frac{w}{2}\right)-a \tan \left(\frac{k_{x}}{\eta} \tan \left(\frac{k_{x} w}{2}\right)\right)\right], & -\infty<x<-\frac{w}{2}\end{cases}
$$




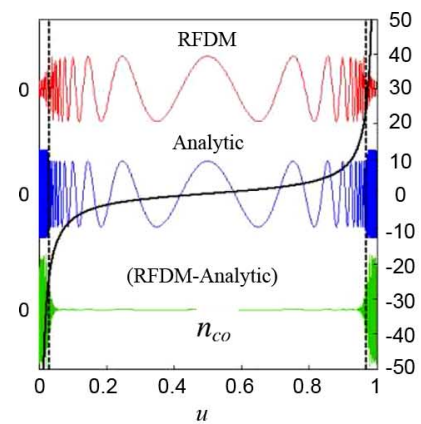

(a)

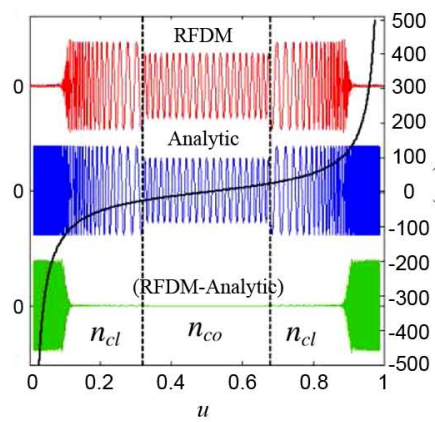

(b)

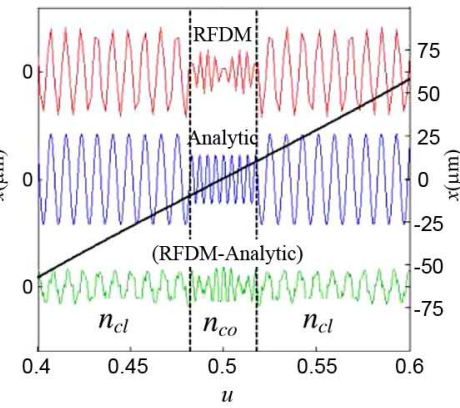

(c)

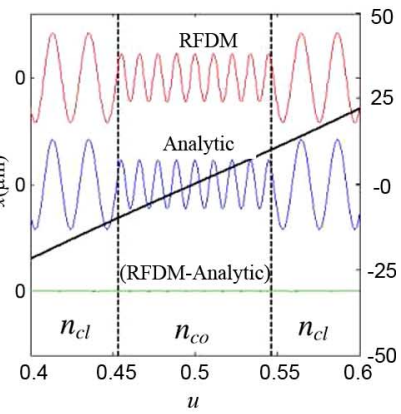

(d)

Fig. 6. Radiation modes calculated by the RFDM method compared to those calculated by an analytic method and the difference between the two showing the error of the RFDM method (a) Even radiation mode in the $u$-domain for $w=50 \mu \mathrm{m}, \beta=11.20 \mu \mathrm{m}^{-1} ; M=256$ and $p=3$ for the RFDM calculated mode that fails to resolve the rapidly oscillating part of the radiation mode close to the waveguide boundaries. (b) Same radiation mode as in (a) but with $p=50$. In this case, RFDM solution accurately converges to the analytic solution inside the waveguide and close to its boundaries. (c) Even radiation mode in the $u$-domain for $w=20 \mu \mathrm{m}, \beta=11.20 \mu \mathrm{m}^{-1} ; M=256$ and $p=200$ for the RFDM obtained solution that fails. (d) Same radiation mode as in (c) but with $p=50$. Again good convergence is observed inside and near the waveguide. Waveguide parameters: $n_{\mathrm{co}}=1.556, n_{\mathrm{cl}}=1.5249$, and $\lambda=850 \mathrm{~nm}$. Dashed lines correspond to the core boundaries in the $u$-domain.

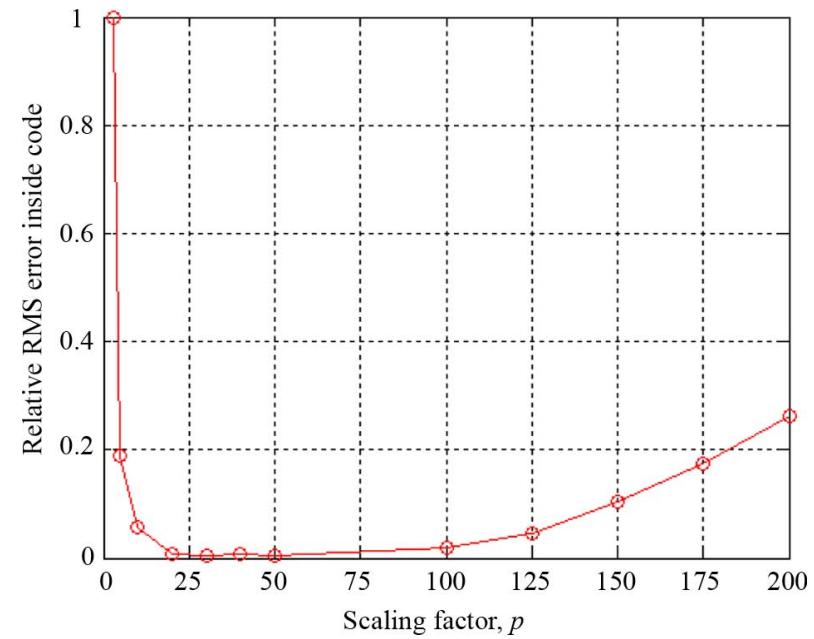

Fig. 7. Relative rms error of (RFDM-analytic) solutions inside the core of a slab waveguide as a function of scaling parameter $p$, and slab dimensions $w=$ $50 \mu \mathrm{m}, n_{\mathrm{co}}=1.5560, n_{\mathrm{cl}}=1.5249$, and $\lambda=850 \mathrm{~nm}, \beta_{r}=11.20 \mu \mathrm{m}^{-1}$, $M=256$

for accurate representation. This can be better understood with the aid of (20). Radiation modes that have propagation constants with $\beta_{r} \cong k n_{\mathrm{cl}}$ have a small value of $k_{x}$, and so oscillate with a low spatial frequency. A small number of Fourier components, $M$, is adequate in this case. On the other hand, the wavenumber $k_{x}$ becomes very large for propagation constants with $\beta \ll k n_{\mathrm{cl}}$, resulting in high-frequency radiation modes. These modes require a larger value of, $M$, in general, to resolve accurately their fine detail. Note that although it is unavoidable to have to increase $M$ to calculate radiation modes with higher spatial frequencies, the number of Fourier components used should be kept to a bare minimum by a judicious choice of scaling parameters in accordance with the recommendations of the previous paragraph. This is because it has been observed that the calculation time increases linearly with $M$ in the case of the slab waveguide and increases quadratically with $M$ and $N$ in the case of the rectangular waveguide.

\section{COUPled POWER THeORY}

In this section, the main aspects of the coupled power theory, adapted specifically for rectangular cross section waveguides,

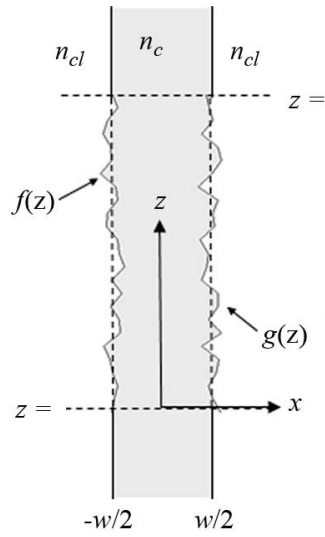

(a)

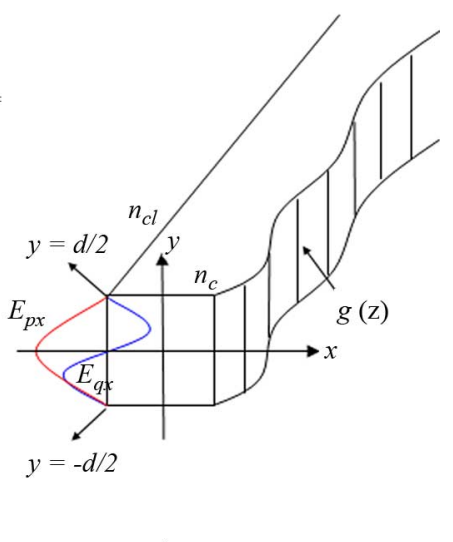

(b)
Fig. 8. (a) Top view of a rectangular waveguide with sidewall roughness (b) Angled view of a randomly deformed waveguide. The 1-D deformation, $g(z)$, affects only the width of the waveguide, while its thickness $d$ remains constant. Also shows the $E_{p x}$ field of the even $p$ th mode in $y$ and $E_{q x}$ field of the even $q$ th mode in $y$, on the waveguide left boundary.

are presented. We refer the reader to see [1]-[8] for a more detailed description of the coupled power theory for slab waveguides and optical fibers. Fig. 8 shows a schematic of a waveguide that will be analyzed. A piece of an imperfect waveguide of length $L$, where $L$ can be infinite, is attached to a perfect waveguide at $z=0$. The refractive index of the perfect waveguide is a function of the transverse coordinates only, $n_{o}=n_{o}(x, y)$. However, when we enter the distorted region, the refractive index varies along the propagation direction $z$ as $n_{f}=n_{f}(x, y, z)$. Specifically, for waveguides fabricated photolithographically, it has been observed [22]-[25] that the sidewall roughness is caused by the roughness of the mask and by the etching pattern. In this case, it is sufficient to assume that the distortion is 1-D only and independent of the $y$-coordinate $\left(\partial n_{f}(x, y, z) / \partial y=0\right)$. In addition, we assume that the distortion function of the left core boundary is a stationary random process described by the function $f(z)$ and that that of the right boundary is also a stationary random process described by the function $g(z)$. The two processes are assumed statistically independent, and so, we can treat them separately. 
If we ignore $g(z)$ for the time being, the power in each of the $Q$ bound waveguide modes is found to be the solution of a system of first-order differential equations according to the coupled power equations [2, p. 184]

$$
\frac{d P_{q}}{d z}=-2 a_{q} P_{q}+\sum_{p=1}^{Q} H_{p q}\left(P_{q}-P_{p}\right)
$$

where $P_{p}$ and $P_{q}$ correspond to the power in the $p$ th and $q$ th modes, respectively, $a_{p}$ is the attenuation coefficient of the $p$ th mode due to coupling between radiation and bound modes, and $H_{p q}$ is the power coupling coefficient between the $p$ th and $q$ th bound modes that is written with the aid of the Wiener-Khinchin theorem as

$$
\begin{aligned}
H_{p q}= & T_{p q}^{2} \int_{-\infty}^{+\infty}\langle f(z) f(z-u)\rangle_{z} \\
& \times \exp \left[-i\left(\beta_{q}-\beta_{p}\right) u\right] d u=T_{p q}^{2}\left|F\left(\beta_{q}-\beta_{p}\right)\right|^{2} .
\end{aligned}
$$

In (22), the \langle\rangle$_{z}$ brackets imply averaging over $z, T_{p q}$ is a constant to be defined later, and $F\left(\beta_{q}-\beta_{p}\right)$ is the Fourier transform of the roughness function $f(z)$ calculated at the difference between the propagation constants $\beta_{p}$ and $\beta_{q}$ of the $p$ th- and $q$ th-coupled modes correspondingly. Usually, Gaussian or exponential distributions are assumed representative of the underlying statistics of the roughness functions. In the Gaussian case, the spectral density function of $f(z)$ becomes, for example,

$$
\left|F\left(\beta_{q}-\beta_{p}\right)\right|^{2}=\sqrt{\pi} S_{d}^{2} L_{c} \exp \left(-\frac{\left(\beta_{q}-\beta_{p}\right)^{2} L_{c}^{2}}{4}\right)
$$

where $L_{c}$ is the autocorrelation length and $S_{d}$ the standard deviation of the random function, $f(z)$.

By substituting the trial solutions $P_{q}=C_{q} e^{-\sigma z}$ in (21), where $C_{q}$ is a constant depending on the amplitude of the $q$ th mode and $\sigma$ is one of the $Q$ eigenvalues of the system, the set of differential (21) is converted to a homogenous system of linear equations. Based on the solutions of these linear equations, the general solution for the average power of the $q$ th mode in the waveguide can be expressed as

$$
P_{q}(z)=\sum_{i=1}^{Q} A_{i} C_{q}^{(i)} \exp \left(-\sigma^{(i)} z\right)
$$

where $A_{i}$ are arbitrary amplitude coefficients $C_{q}^{(i)}, i=$ $1, \ldots, Q$, are the $Q$ orthogonal eigenvectors corresponding to the $q$ th mode and $\sigma^{(i)}, i=1, \ldots, Q$, are the $Q$ eigenvalues of the system, not necessary all different, which are calculated from the system's determinant. Each eigenvector $C_{q}^{(i)}$ in (24) is calculated by solving the homogenous system for the $i$ th eigenvalue $\sigma^{(i)}$. Finally, the solution is completed by calculating the amplitude coefficients $A_{i}$. This calculation is carried out using (24) and by taking advantage of the orthogonality condition of the eigenvectors at $z=0$ to give

$$
A_{i}=\sum_{i=1}^{Q} C_{q}^{(i)} P_{q}(0)
$$

Equation (25) implies that the coefficients $A_{i}$ are expressed as a linear combination of the powers of the $Q$ bound modes at the beginning of the waveguide where the power distribution is assumed to be known.

If the eigenvalues are arranged in ascending order as $\sigma^{(1)} \sigma^{(2)} \leq \cdots \leq \sigma^{(M)}$ then the ratio between the first term and the second term in (24) is

$$
R=\exp \left(\sigma^{(2)}-\sigma^{(1)}\right) z .
$$

This ratio monotonically increases with $z$. Therefore, for large $z$, only the first term in (24) is important and dominates over the rest of the eigenvectors. The implication of the previous equation is that the distribution of power between modes ultimately becomes independent of the initial power in the modes and converges to a constant distribution determined by the first eigenvector $A_{1}$ only. The distance $L_{\text {eq }}$ required for the power distribution to reach this equilibrium can be calculated from (26) to be

$$
L_{\mathrm{eq}}=\frac{(\ln R)}{\left(\sigma^{(2)}-\sigma^{(1)}\right)} .
$$

Equation (27) implies that equilibrium is reached when the largest term of the general solution (24) is $R$ times larger than the second largest term. In most applications, $R=100$ is a reasonable estimate.

Let us now consider the evolution of the modal power distribution after equilibrium has been reached. Since the first eigenvector is the dominant one, the power distribution propagates as

$$
P_{\mathrm{eq}}(z)=\left[\sum_{q=1}^{Q} C_{q}^{(1)} P_{\mathrm{eq}}(0)\right] C_{q}^{(1)} \exp \left(-\sigma^{(1)} z\right) .
$$

According to (28), power is lost at equilibrium at a rate that depends on the first eigenvalue $\sigma^{(1)}$ only. As a consequence, the eigenvalue $\sigma^{(1)}$ corresponds to the waveguide average propagation loss rate due to scattering of bound modes to radiation modes.

\section{A. Derivation of Coupling Coefficients}

In order to implement the coupled power theory, it is necessary to calculate the coupling coefficients between the bound modes and to calculate the attenuation coefficients of the bound modes due to coupling to the continuum of radiation modes. According to [2, p. 106], the coupling coefficient $h_{p q}$ between the $p$ th and $q$ th forward propagating bound modes is expressed in an integral form involving the transverse and longitudinal electric field components of each mode as

$$
\begin{aligned}
h_{p q}=\frac{\omega \varepsilon_{o}}{4 i}\{ & \int_{-\infty}^{+\infty} \int_{-\infty}^{+\infty}\left(n_{f}^{2}-n_{o}^{2}\right)\left[\mathbf{E}_{p x}^{*} \cdot \mathbf{E}_{q x}\right. \\
& \left.\left.+\mathbf{E}_{p y}^{*} \cdot \mathbf{E}_{q y}+\left(\frac{n_{o}^{2}}{n_{f}^{2}}\right) \mathbf{E}_{p z}^{*} \cdot \mathbf{E}_{q z}\right] d x d y\right\}
\end{aligned}
$$

Both modes in (29) are normalized to unit power flow. By applying the dielectric boundary conditions for the transverse and longitudinal field components at $x=w / 2$ and by taking into account that $\left(n_{f}^{2}-n_{o}^{2}\right)=0$ everywhere except at $x=w / 2$, and 
that $E_{y}=0$ for the $E_{x}$-type modes in the rectangular waveguide, (29) reduces for rectangular waveguides to

$$
\begin{aligned}
h_{p q}=\frac{\omega \varepsilon_{o}}{4 i}\left\{\int_{-d / 2}^{d / 2}\right. & \left(n_{\mathrm{cl}}^{2}-n_{\mathrm{co}}^{2}\right) f(z)\left[\left(\frac{n_{\mathrm{cl}}^{2}}{n_{\mathrm{co}}^{2}}\right)\right. \\
& \left.\left.\times \mathbf{E}_{p x}^{*} \cdot \mathbf{E}_{q x}+\mathbf{E}_{p z}^{*} \cdot \mathbf{E}_{q z}\right]_{x=w / 2} d y\right\}
\end{aligned}
$$

or equivalently

$$
h_{p q}=T_{p q} f(z)
$$

where $T_{p q}$ is the constant first introduced in (22) and is now seen to be

$$
\begin{aligned}
& T_{p q}=\frac{\omega \varepsilon_{o}}{4 i} \int_{-d / 2}^{d / 2}\left(n_{\mathrm{cl}}^{2}-n_{\mathrm{co}}^{2}\right)\left[\left(\frac{n_{\mathrm{cl}}^{2}}{n_{\mathrm{co}}^{2}}\right)\right. \\
&\left.\times \mathbf{E}_{p x}^{*} \cdot \mathbf{E}_{q x}+\mathbf{E}_{p z}^{*} \cdot \mathbf{E}_{q z}\right]_{x=w / 2} d y .
\end{aligned}
$$

The power coupling coefficients can now be calculated with the aid of (22) and (23). Careful study of (30)-(32) leads to a very important conclusion. The coupling coefficient $h_{p q}$ between any two bound modes is proportional to the product of their electric field values taken on the waveguide boundary and then integrated in the direction of its thickness. However, if the two modes have different symmetries in the direction of the waveguide thickness, for example the $p$ th mode has even symmetry in $y$ and the $q$ th mode has odd symmetry in $y$ [see Fig. 8(b)], then their product is an odd function whose integral along a symmetrical interval about $y=0$ is exactly zero. According to the modal symmetries defined in Section II, the modes with even-even and odd-even symmetry only couple with the modes of the same symmetry group (even-even and odd-even). Similarly, modes with odd-odd and even-odd symmetry can only couple to odd-odd and even-odd modes only. The presence of two uncoupled sets of modes implies that two independent modal distributions survive at equilibrium for rectangular waveguides with 1-D sidewall roughness.

\section{B. Attenuation Coefficients Due to Coupling of Bound to Radiation Modes}

Equations (30)-(32) can be used to express the power coupling coefficient between any two modes although, in the previous section, special reference was made to coupling between bound modes. If one mode were a radiation one, then the same equation would still hold, but this time it would describe the power coupling between a bound and a radiation mode. The only difference is that radiation modes are normalized to an impulse power flow. Therefore, similarly to (22), if the $p$ th bound mode is replaced with the $r$ th radiation mode, the power coupling coefficient $J_{p r}$ between the $p$ th bound and the $r$ th radiation mode is given by

$$
J_{q r}=T_{q r}^{2}\left|F\left(\beta_{q}-\beta_{r}\right)\right|^{2}
$$

with $T_{q r}$ as in (32) with $p \rightarrow r$. If we assume that the power coupled from bound to radiation modes is lost, then the attenuation coefficient $a_{q}$ of the $q$ th guided mode is given by integrating over all radiation modes [2, p. 116]

$$
2 a_{q}=\sum_{4} \int_{0}^{k n_{\mathrm{cl}}}\left|T_{q r}\right|^{2}\left|F\left(\beta_{r}-\beta_{q}\right)\right|^{2}\left(\frac{\left|\beta_{r}\right|}{r}\right) d \beta_{r}
$$

where $r$ is the transverse wavenumber of the $r$ th radiation mode (18). The $\Sigma$ symbol in (34) implies summation over the four different sets of symmetries of the radiation modes. We note that similar arguments as in the case of the bound-to-bound mode coupling, suggest that bound modes of a specific symmetry group can lose energy due to coupling to radiation modes of the same symmetry group only.

\section{ApPlication of the Coupled Power Theory to ReCTANGUlar CROSS SECTION WAVEGUIDES}

In this section, the coupled power theory is applied to a multimode rectangular cross section waveguide (see Fig. 1) with parameters $w=d=50 \mu \mathrm{m}, \lambda=850 \mathrm{~nm}, n_{\mathrm{cl}}=1.5560$, and $n_{\text {co }}=1.5249$ used in optical backplane interconnection applications [28]. The aim here is to calculate the power distribution of the modes at equilibrium, the steady-state propagation loss and, finally, the propagation distance at which equilibrium is reached. Based on measurements by atomic force microscopy (AFM), the sidewalls of a waveguide were found to have a roughness with standard deviations varying in the range $10 \mathrm{~nm}<\sigma<100 \mathrm{~nm}$ and autocorrelation lengths varying in the range $4 \mu \mathrm{m}<L_{c}<10 \mu \mathrm{m}$. Bound modes of the waveguide were calculated using the FDM method [21] and a total of 1052 nondegenerate bound modes were found. Radiation modes were calculated with the RFDM method introduced in Section II.

\section{A. Segmentation of Radiation Modes}

The attenuation of any propagating bound mode is calculated by integrating over the continuum of radiation mode propagation constants [see (34)]. Since RFDM is semianalytic, the radiation-mode spectrum has to be segmented and the integrations performed numerically. The segmentation follows from the definition of the transverse wavenumber $r$ [see (18)]. The locus of $k$ is a circle with radius $r$ and we can write

$$
k_{x}=r \cos \theta, \quad k_{y}=r \sin \theta
$$

with $0<\theta<\pi / 2$ and $0<r<k n_{\mathrm{cl}}$, for the forward propagating free-space modes in (17a)-(17d). In order to calculate all free-space modes, and hence, all radiation modes, we simply need to scan along the two parameters, the angle $\theta$ and the transverse wavenumber $r$, between their limiting values. In fact, we can confine our calculations to the vicinity of $k n_{\mathrm{cl}}$ only for the transverse wavenumbers $r$. This is because the power coupling coefficients between bound and radiation modes [see (33)] are proportional to the spectral density function of the waveguide roughness [see (23)], which decreases rapidly as the radiation mode propagation constant $\beta_{r}$ decreases. If we assume that the propagation constant of the last bound mode (closest to cutoff) is $\beta_{q} \cong k n_{\mathrm{cl}}$, and we assume that there are no contributions to 

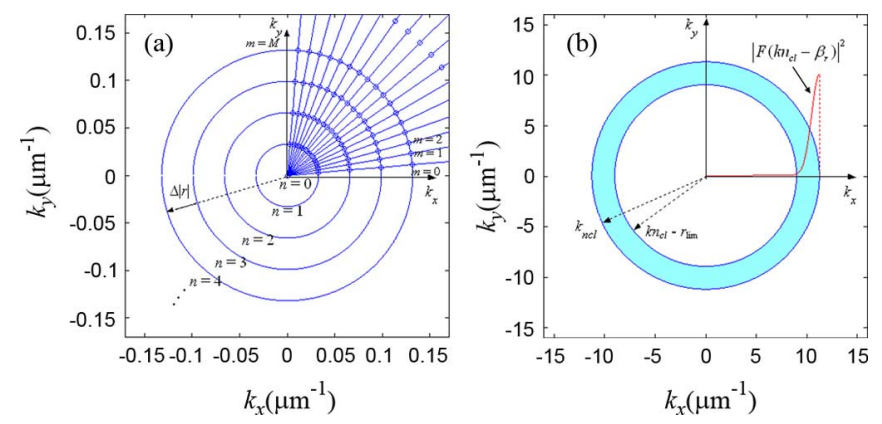

Fig. 9. (a) Constellation diagram of $k_{x}$ and $k_{y}$ wavenumbers used to discretize the free-space mode spectrum. (b) Although $0<r<k n_{\mathrm{cl}}$, only the thin annulus with $k n_{\mathrm{cl}}-r_{\mathrm{lim}}<r<k n_{\mathrm{cl}}$ is necessary for the bound-to-radiation coupling and attenuation coefficient calculations.

the power coupling coefficients (33) when the spectral density function has dropped by more than $10^{-3}$ of its maximum value, then for Gaussian statistics, we find from (23) an upper limit $\beta_{\lim }$ for $\beta_{r}$

$$
\beta_{\lim }=\beta_{\lim }\left(L_{c}\right)=\sqrt{\frac{-4 \log (0.001)}{L_{c}^{2}}}=\frac{5.256}{L_{c}} .
$$

We can therefore assume that $\beta_{r}$ and $r$ are now confined within the new regions

$$
0<\beta_{r}<\beta_{\text {lim }}, \quad k n_{\mathrm{cl}}-\beta_{\lim }<r<k n_{\mathrm{cl}} .
$$

The two free-space-mode parameters $\theta$ and $r$ are next segmented into $K$ and $L$ discrete steps, respectively (see Fig. 9), as

$$
\begin{aligned}
& \theta=k \Delta \theta, \quad k=0, \ldots, K, \quad \Delta \theta=\frac{\pi}{2 K} \\
& r=k n_{\mathrm{cl}}-l \Delta r, \quad l=0, \ldots, L, \quad \Delta r=\frac{\beta_{\mathrm{lim}}}{L} .
\end{aligned}
$$

Initial simulation tests revealed $K=18$ to be a reasonable choice, giving $\Delta \theta=5^{\circ}$. These choices assured convergence of the attenuation coefficients to four decimal points (in decibels per centimeter). Based on the AFM measurements, the worst autocorrelation length $L_{c}=4 \mu \mathrm{m}$ (36) and (37) gives $r_{\text {lim }}=1.32 \mu \mathrm{m}^{-1}$. Based on our tests we chose $L=40$, thus giving $\Delta r=0.033$. With these values, a total of 2880 radiation modes were generated. In order to normalize the radiation modes, we only had to normalize their free-space part, as suggested in [14]and [27]. This normalization step is carried out in Appendix B.

\section{B. Computation of Radiation Modes}

In order to achieve convergence of the RFDM algorithm, a Fourier series with $M=N=192$ components was used. After quantization, this yielded a linear system of equations with $192^{2} \times 192^{2}$ unknowns. Memory constraints prohibited the solution of this system on a single computer. Thus, a parallel solver was employed. In order to solve the system of linear equations (12), we used the scaLAPACK library, specifically the PDGESV routine, which is based on an LU decomposition method [29]. Each radiation mode required $\sim 17$ min to calculate on a $4 \times 4$ processor grid array comprising nodes equipped with $900 \mathrm{MHz}$ processors and 4 GB RAM.

\section{Mode Propagation in $50 \mu \mathrm{m} \times 50 \mu \mathrm{m}$ Waveguides}

First, we examine the effect of the waveguide sidewall roughness standard deviation on the equilibrium loss and equilibrium length. This is shown in Fig. 10(a) for a waveguide whose roughness autocorrelation length has been set to $L_{c}=4 \mu \mathrm{m}$ and its standard deviation varied in the range $10 \mathrm{~nm}<S_{d}<100 \mathrm{~nm}$. As mentioned previously, two independent distributions coexist at equilibrium, one for each set of symmetries. The equilibrium loss and length for the even-even and odd-even ensemble are shown with circles in Fig. 10(a), while those for the odd-odd, even-odd ensemble are shown with rectangles. We also show with triangles the equilibrium loss and length for a slab waveguide with the same physical parameters $\left(w, n_{\mathrm{cl}}, n_{\mathrm{co}}, S_{d}, L_{c}\right)$ as the square waveguide under investigation, for comparison purposes.

As the standard deviation increases, we observe that the equilibrium loss increases for both symmetries in Fig. 10(a). A larger standard deviation corresponds to a waveguide with "rougher" sidewalls, which, in turn, causes bound modes to be coupled more strongly to the radiation modes. Therefore, a larger propagation loss is expected at equilibrium. In addition, we observe that the length, required to reach the equilibrium follows an opposite trend and decreases. This is because, not only does the magnitude of the bound-to-radiation coupling coefficients increase, but also the magnitude of the bound-to-bound coupling coefficients increases. As bound modes are coupled more strongly to each other, they exchange energy at faster rates and equilibrium is reached in shorter lengths. Compared to the square waveguide, a slab waveguide with similar properties has a lower steady-state loss and a longer equilibrium length. However, the results for the slab waveguide are of the same order of magnitude as those for the square waveguide. Therefore, a slab waveguide can be used in cases when a fast but relatively inaccurate approximation for the propagation loss and length is required. The steady-state loss for the odd-odd, even-odd ensemble is slightly higher than that of the even-even and odd-even ensemble. It is not yet clear why this is the case.

As a particular example, a waveguide with autocorrelation length $L_{c}=4 \mu \mathrm{m}$ and standard deviation of sidewall roughness $S_{d}=30 \mathrm{~nm}$, has a steady-state loss for the even-even, odd-even modes of $a_{\text {eeoe }}=0.0008 \mathrm{~dB} / \mathrm{cm}$ and equilibrium length $L_{\text {eeoe }}=43.1 \mathrm{~m}$. The odd-odd and even-odd modes have a steady-state loss of $a_{\text {ooeo }}=0.0013 \mathrm{~dB} / \mathrm{cm}$, and equilibrium length $L_{\text {ooeo }}=34.7 \mathrm{~m}$. Only 1 mode out of the 537 modes belonging in the even-even/odd-even symmetry ensemble [see Fig. 11(a)], and 1 mode out of the 515 modes belonging in the odd-odd/even-odd ensemble [see Fig. 11(b)] survive at equilibrium. Fig. 11(a) shows that the mode that survives from the even-even/odd-even symmetry ensemble is the fundamental mode of the waveguide with $\beta=11.5016 \mu \mathrm{m}^{-1}$. Similarly, Fig. 11(b) shows the mode that survives from the odd-odd/even-odd symmetry ensemble is the first even-odd mode with $\beta=11.5011 \mu \mathrm{m}^{-1}$. Therefore, our theory predicts dual-mode operation at equilibrium for the specific example. In fact, it can be shown that dual-mode operation is expected for all autocorrelation lengths and roughness standard deviations 


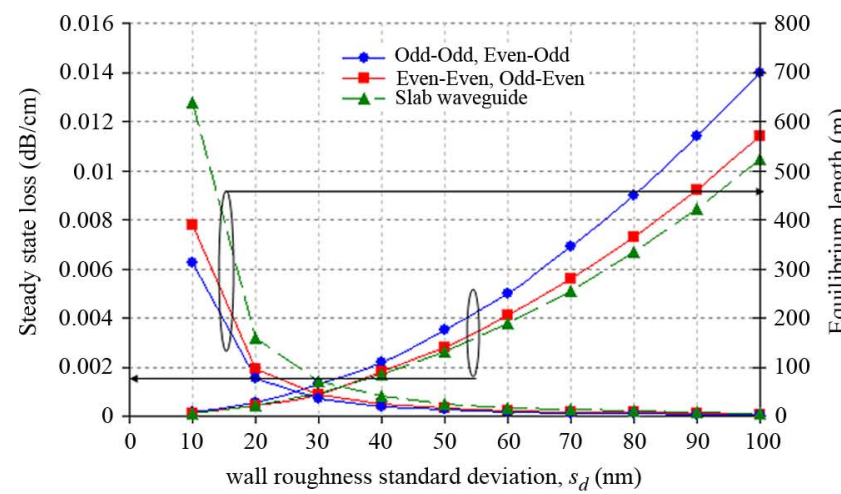

(a)

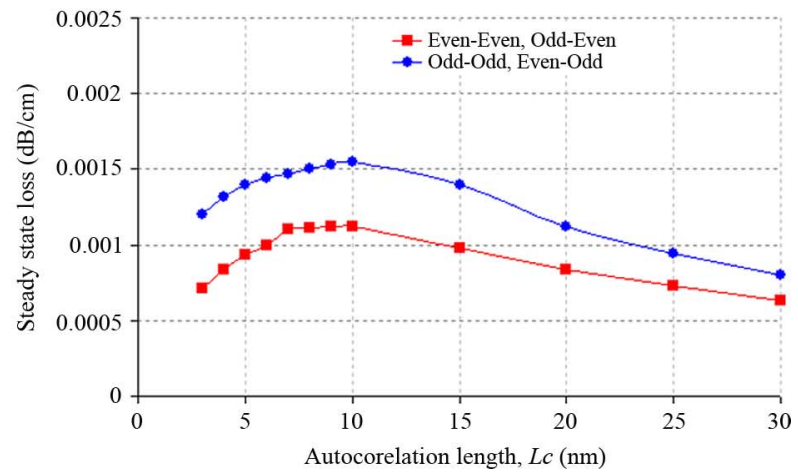

(b)

Fig. 10. (a) Steady-state loss and equilibrium length as a function of the sidewall roughness standard deviation for $L_{c}=4 \mu \mathrm{m}$. (b) Steady-state loss as a function of the roughness autocorrelation length for $S_{d}=25 \mathrm{~nm}$.

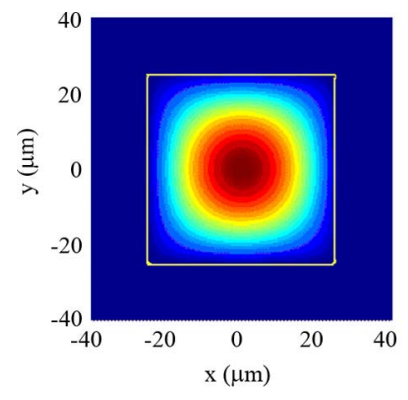

(a)

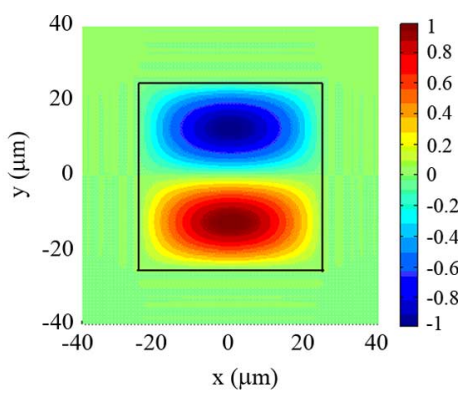

(b)
Fig. 11. Modes surviving at equilibrium for a $50 \mu \mathrm{m} \times 50 \mu \mathrm{m}$ square waveguide having sidewall roughness with statistical parameters, standard deviation, $S_{d}=30 \mathrm{~nm}$, autocorrelation length, $L_{c}=4 \mu \mathrm{m}$. (a) Fundamental waveguide mode surviving from the even-even, even-odd mode ensemble. (b) First even-odd mode surviving from the odd-odd, even-odd mode ensemble.

examined in this section. A roughness with much smaller autocorrelation length that would couple bound modes much stronger with each other would be required to allow the survival of a larger set of modes at equilibrium.

The next set of calculations examine the effect of the autocorrelation length of the roughness. The standard deviation is set to $S_{d}=30 \mathrm{~nm}$ and the autocorrelation length is varied over the range, $3 \mu \mathrm{m}<L_{c}<10 \mu \mathrm{m}$. Fig. 10(b) shows that the steady-state loss increases as the autocorrelation length of the sidewall roughness increases until $L_{c} \sim 10 \mu \mathrm{m}$, when the steady-state loss begins to decrease. These results are similar to the results for optical fibers and slab waveguides in [2, p. 187] where it was reported that the steady-state loss reaches a maximum value when the radius of the fiber (or the width of the slab) was approximately $0.3-0.4$ times smaller than the autocorrelation length. For square cross section of $50 \mu \mathrm{m} \times 50 \mu \mathrm{m}$ waveguides with 1-D sidewall roughness, we find that the steady-state loss reaches a maximum value when the width of the waveguide is 0.2 times smaller than the autocorrelation length. It is, therefore, advisable to avoid fabricating waveguides with widths, thicknesses, and autocorrelation lengths satisfying this relationship, if possible, to avoid the maximum propagation loss.

\section{CONCLUSION}

We introduced RFDM, a new semianalytic method to construct radiation modes in rectangular cross section waveguides and used coupled power theory to describe mode propagation in waveguides with random sidewall roughness. The calculated coupling coefficients between bound-to-bound and bound-to-radiation modes showed that modes that have different symmetry along the waveguide thickness direction, cannot interact with each other in the presence of 1-D sidewall roughness. Therefore, two sets of modes propagate independently from each other inside the waveguide and gradually converge to two uncorrelated distributions at equilibrium. Investigation of the propagation properties of $50 \mu \mathrm{m} \times 50 \mu \mathrm{m}$ waveguides, typically used in optical backplane interconnections, showed that the equilibrium loss reaches a maximum value when the width of the waveguide was about five times the autocorrelation length of the sidewall roughness. It was also shown that equilibrium lengths were much larger than the length of any backplane. There is a difficulty in assigning a unique propagation loss to a waveguide as the loss at the start of the waveguide is high due to loss of more radiation modes and gradually reduces up to the equilibrium length of $>30 \mathrm{~m}$. The results also imply that the experimentally measured loss depends on the excitation conditions.

\section{APPENDIX A}

The matrices in (15a)-(15d) take the following analytic form for the odd-odd case:

$$
\begin{aligned}
\left(\frac{\partial u}{\partial x}\right)^{2}= & \frac{1}{8 \pi^{2} a_{x}^{2}}[3-4 \cos (2 \pi u)+\cos (4 \pi u)] \\
\frac{\partial^{2} u}{\partial x^{2}}= & \frac{1}{2 \pi a_{x}^{2}}[3 \sin (\pi u)-\sin (3 \pi u)] \\
\left(\frac{\partial v}{\partial y}\right)^{2}= & \frac{1}{8 \pi^{2} a_{y}^{2}}[3-4 \cos (2 \pi v)+\cos (4 \pi v)] \\
\frac{\partial^{2} v}{\partial y^{2}}= & \frac{1}{2 \pi a_{y}^{2}}[3 \sin (\pi v)-\sin (3 \pi v)] \\
\mathbf{I}_{1}^{\text {oo }}= & -l^{2} \pi^{2} \int_{0}^{1} \int_{0}^{1}\left(\frac{\partial u}{\partial x}\right)^{2} S_{l m}^{\mathrm{oo}}(u, v) S_{l^{\prime} m^{\prime}}^{\mathrm{oo}}(u, v) d u d v \\
= & \frac{m^{2}}{a_{x}^{2}}\left\{\frac{3}{2} \delta_{l, l^{\prime}}-\delta_{l, l^{\prime}+1}-\delta_{l, l^{\prime}-1}\right. \\
& \left.+\frac{1}{4} \delta_{l, l^{\prime}+2}+\frac{1}{4} \delta_{l, l^{\prime}-2}-\frac{1}{4} \delta_{l, 2-l^{\prime}}\right\} \delta_{m, l^{\prime}}
\end{aligned}
$$




$$
\begin{aligned}
\mathbf{I}_{2}^{\mathrm{Oo}}= & l \pi \int_{0}^{1} \int_{0}^{1} \frac{\partial^{2} u}{\partial x^{2}} \frac{1}{\tan (l \pi u)} S_{l m}^{\mathrm{oo}}(u, v) \\
& \times S_{l^{\prime} m^{\prime}}^{\mathrm{Oo}}(u, v) d u d v \\
= & \frac{l}{2 a_{x}^{2}}\left\{\delta_{l, l^{\prime}+1}-\delta_{l, l^{\prime}-1}-\frac{1}{2} \delta_{l, l^{\prime}+2}+\frac{1}{2} \delta_{l, l^{\prime}-2}\right. \\
& \left.+\frac{1}{2} \delta_{l, 2-l^{\prime}}\right\} \delta_{m, m^{\prime}} .
\end{aligned}
$$

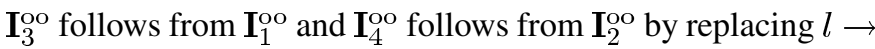
$m$ and $\alpha_{x} \rightarrow \alpha_{y}$. Similar results are obtained for the matrices for the other symmetries.

\section{APPENDIX A}

In this appendix, we present the normalization of the freespace modes of (17a)-(17d). We start by calculating all field components of the modes. We follow Marcatilli's approach [26] for the field representation, which assumes $E_{y}=0$ for the $E_{x}$ polarized modes, and express all field components as a function of the transverse components

$$
\begin{aligned}
H_{x} & =\frac{1}{\omega^{2} \mu_{o} \varepsilon_{o} n^{2}(x, y)} \frac{\partial^{2} E_{x}}{\partial y^{2}} \\
H_{y} & =-\frac{1}{\omega^{2} \mu_{o} \varepsilon_{o} n^{2}(x, y)} \frac{\partial^{2} E_{x}}{\partial x \partial y}-\frac{\beta_{r}}{\omega \mu_{o}} E_{x} \\
H_{z} & =\frac{i}{\omega \mu_{o}} \frac{\partial E_{x}}{\partial y} \\
E_{z} & =-\frac{i}{\omega \mu_{o}}\left(\frac{\partial H_{x}}{\partial y}+\frac{\partial H_{y}}{\partial x}\right)
\end{aligned}
$$

with $\mu_{o}$, the magnetic permeability, $\varepsilon_{o}$, the vacuum dielectric constant, and $\omega$, the angular frequency of light. The even-even free-space radiation mode whose $E_{x}$ component is given in (17a) has the following transverse field components, for example

$$
\begin{aligned}
E_{x}^{f, \mathrm{ee}}= & A^{\mathrm{ee}} \cos \left(k_{x} x\right) \cos \left(k_{y} y\right) \\
H_{x}^{f, \mathrm{ee}}= & \frac{A^{\mathrm{ee}} k_{x} k_{y}}{k^{2} n_{\mathrm{cl}}^{2}} \sin \left(k_{x} x\right) \cos \left(k_{y} y\right) \\
H_{y}^{f, \mathrm{ee}}= & A^{\mathrm{ee}}\left(\frac{k_{x} k_{y}}{k^{2} n_{\mathrm{cl}}^{2}} \sin \left(k_{x} x\right) \cos \left(k_{y} y\right)\right. \\
& \left.-\frac{\beta_{r}}{\omega \mu_{o}} \cos \left(k_{x} x\right) \cos \left(k_{y} y\right)\right) .
\end{aligned}
$$

To calculate the power flow through a waveguide cross-section, we need the Poynting vector

$$
S_{z}=\frac{1}{2} \operatorname{Re}\left(\mathbf{E} \times \mathbf{H}^{*}\right)_{z} .
$$

The power flow $P$ through a plane perpendicular to the direction of propagation is found by substituting (50)-(52) into (53) and integrating over an infinite cross section to give

$$
P=-\frac{1}{2} \int_{-\infty}^{+\infty} \int_{-\infty}^{+\infty} S_{z} d x d y=-\frac{1}{2} \int_{-\infty}^{+\infty} \int_{-\infty}^{+\infty} E_{x} H_{y}^{*} d x d y
$$

$$
\begin{aligned}
P= & \frac{1}{2} \frac{\beta_{r}\left(A^{\mathrm{ee}}\right)^{2}}{\omega \mu_{o}} \cdot \int_{0}^{+\infty} \int_{0}^{+\infty} \cos \left(\left(k_{x}-k_{x}^{\prime}\right) x\right) \\
& \times \cos \left(\left(k_{y}-k_{y}^{\prime}\right) y\right) d x d y \\
= & \frac{\pi^{2}}{2} \frac{\beta_{r}\left(A^{\mathrm{ee}}\right)^{2}}{\omega \mu_{o}} \cdot \delta\left(\left(k_{x}-k_{x}^{\prime}\right) x\right) \delta\left(\left(k_{y}-k_{y}^{\prime}\right) y\right)
\end{aligned}
$$

where $\delta$ is the Dirac delta function in (55). For unit power flow $P$ we have from (55)

$$
A^{\mathrm{ee}}=\left[\frac{2}{\pi^{2}} \frac{\omega \varepsilon_{o}}{\beta_{r}}\right]^{1 / 2} .
$$

The normalization of the other radiation modes is the same

$$
A^{\mathrm{ee}}=A^{\mathrm{eo}}=A^{\mathrm{oe}}=A^{\mathrm{oo}}=\left[\frac{2}{\pi^{2}} \frac{\omega \varepsilon_{o}}{\beta_{r}}\right]^{1 / 2}
$$

\section{ACKNOWLEDGMENT}

The authors thank Dr. J. Yates and UCL research computing services for making available the Keter SUN cluster for parallel computing calculations. They also thank D. Milward, K. Hopkins, and R. Pitwon, Xyratex Technology Ltd., Navin Suyal Exxelis Ltd., and Frank Tooley, ITI Techmedia for fruitful discussions.

\section{REFERENCES}

[1] D. Marcuse, Light Transmission Optics. London, UK: Van Nostrand Reinhold, 1972.

[2] D. Marcuse, Theory of Dielectric Waveguides. London, UK: Academic, 1991.

[3] D. Marcuse, "Radiation losses of dielectric waveguides in terms of the power spectrum of the wall distortion function," Bell Syst. Tech. J., vol. 48, pp. 3233-3243, Dec. 1969.

[4] D. Marcuse, "Mode conversion caused by surface imperfections of a dielectric slab waveguide," Bell Syst. Tech. J., vol. 48, pp. 3186-3214, Dec. 1969.

[5] D. Marcuse, "Derivation of coupled power equations," Bell Syst. Tech. J., vol. 51, pp. 229-237, Jan. 1972.

[6] D. Marcuse, "Power distribution and radiation losses in multimode dielectric slab waveguides," Bell Syst. Tech. J., vol. 51, pp. 429-454, Feb. 1972.

[7] D. Marcuse, "Higher-order scattering losses in dielectric waveguides," Bell Syst. Tech. J., vol. 51, pp. 1801-1817, Oct. 1972.

[8] D. Marcuse, "Higher-order loss processes and the loss penalty of multimode operation," Bell Syst. Tech. J., vol. 51, pp. 1819-1836, Oct. 1972.

[9] K. K. Lee, D. R. Lim, H.-C. Luan, A. Agarwal, J. Foresi, and L. C. Kimerling, "Effect of size and roughness on light-transmission in a $\mathrm{Si} / \mathrm{SiO}_{2}$ waveguide: Experiments and model," Appl. Phys. Lett., vol. 77, pp. 1617-1619, 2000.

[10] F. Grillot, L. Vivien, S. Laval, D. Pascal, and E. Cassan, "Size influence on the propagation loss induced by sidewall roughness in ultrasmall SOI waveguides," IEEE Photon. Technol. Lett., vol. 16, no. 7, pp. 1661-1663, Jul. 2004.

[11] D. Lenz, D. Erni, and W. Bächtold, "Modal power loss coefficients for highly overmoded rectangular dielectric waveguides based on free space modes," Opt. Express, vol. 12, pp. 1150-1156, Mar. 2004.

[12] M. Reed, T. M. Benson, P. Sewell, P. C. Kendall, G. M. Berry, and S. V. Dewar, "Free space radiation mode analysis of rectangular dielectric waveguides," Opt. Quantum. Elctron., vol. 28, pp. 1175-1179, 1996.

[13] Z. H. Wang, "Free space mode approximation of radiation modes for weakly guiding planar optical waveguides," IEEE J. Quantum Electron., vol. 34, no. 4, pp. 680-685, Apr. 1998.

[14] C. G. Poulton, C. Koos, M. Fujii, A. Pfrang, T. Schimmel, J. Leuthold, and W. Freude, "Radiation modes and roughness loss in high indexcontrast waveguides," IEEE J. Sel. Top. Quantum Electron., vol. 12, no. 6, pp. 1306-1320, Nov./Dec. 2006.

[15] S. G. Johnson, M. L. Povinelli, M. Soljacic, A. Karalis, S. Jacobs, and J. D. Joannopolous, "Roughness losses and volume-current methods in photonic-crystal waveguides," Appl. Phys. B, vol. 81, pp. 283-293, 2005. 
[16] M. Kuznetsov and H. A. Haus, "Radiation loss in dielectric waveguide structures by the volume current method," IEEE J. Quantum Electron., vol. QE-19, no. 10, pp. 1505-1514, Oct. 1983.

[17] T. Barwicz and H. A. Haus, "Three-dimensional analysis of scattering losses due to sidewall roughness in microphotonic waveguides," $J$. Lightwave Technol., vol. 23, no. 9, pp. 2719-2732, Sep. 2005.

[18] J. P. R. Lacey and F. P. Payne, "Radiation loss from planar waveguides with random wall imperfections," Proc. Inst. Electr. Eng., vol. 137, pp. 282-288, Aug. 1990.

[19] T. Bierhoff, A. Wallrabenstein, A. Himmler, E. Griese, and G. Mrozynski, "Ray tracing technique and its verification for the analysis of highly multimode optical waveguides with rough surfaces," IEEE Trans. Magnetics, vol. 37, no. 5, pp. 3307-3310, Sep. 2001.

[20] C. H. Henry and B. H. Verbeek, "Solution of the scalar wave equation for arbitrarily shaped dielectric waveguides by two-dimensional Fourier analysis," J. Lightwave Technol., vol. 7, no. 2, pp. 308-383, Feb. 1989.

[21] S. J. Hewlett and F. Ladoucer, "Fourier decomposition method applied to mapped infinite waveguides: Scalar analysis of dielectric waveguides down to modal cutoff," J. Lightwave Technol., vol. 13, no. 3, pp. 375-383, Mar. 1995.

[22] F. Ladoucer, J. D. Love, and T. J. Senden, "Measurement of surface roughness in burried channel waveguides," Electron. Lett., vol. 28, pp. 1321-1322, Jul. 1992.

[23] F. Ladoucer, J. D. Love, and T. J. Senden, "Effect of side wall roughness in buried channel waveguides," Inst. Electr. Eng. Proc. Optoelectron., vol. 141, pp. 242-248, Aug. 1994.

[24] F. Ladoucer and L. Poladian, "Surface roughness and backscattering," Opt. Lett., vol. 21, pp. 1833-1835, Nov. 1996.

[25] F. Ladoucer, "Roughness, inhomogeneity, and integrated optics," $J$. Lightwave Technol., vol. 15, no. 6, pp. 1020-1025, Jun. 1997.

[26] E. A. J. Marcatili, "Dielectric rectangular waveguide and directional coupler for integrated optics," Bell Syst. Tech. J., vol. 48, pp. 2071-2101, Dec. 1969.

[27] R. A. Sammut, "Orthogonality and normalization of radiation modes in dielectric waveguides," J. Opt. Soc. Am., vol. 72, pp. 1335-1337, Oct. 1982.

[28] I. Papakonstantinou, D. R. Selviah, R. Pitwon, and D. Milward, "Low cost precision, self-alignment technique for coupling lasers and photodiode arrays to polymer waveguide arrays on multilayer PCBS," IEEE Trans. Adv. Packag., vol. 31, no. 3, pp. 502-511, Aug. 2008.

[29] G. H. Golub and C. F. VanLoan, Matrix Computations, 3rd ed. Baltimore, MD: The Johns Hopkins University Press, 1996.

Ioannis Papakonstantinou (S'02-M'09) graduated in electrical and computer engineering from the National Technical University of Athens (NTUA), Athens, Greece, in 2000, the M.Sc. degree (with distinction) in broadband technologies for communications and the Ph.D. degree in optical backplane interconnections from the University College London (UCL), London, U.K., in 2002 and 2008, respectively.

For one year he was with Sharp Laboratories of Europe investigating diffraction gratings for multifunctional mobile phone backlights. In 2009 he joined CERN-European Organization for Nuclear Research, Geneva, Switzerland. $\mathrm{He}$ is currently engaged in research on passive optical networks for the distribution of timing-trigger-control signals in the super large Hadron collider (S-LHC). He is the author or coauthor of about 20 papers in journals and conference proceedings, and the inventor or coinventor of two granted and five pending patents.

Dr. Papakonstantinou is a Graduate Member of the Optical Society of America, an IEEE, and OSA reviewer, and since 2007, he has been a member of the Program Committee of the IEEE Workshop on Interconnections Within High-Speed Digital Systems (HSDS). He received an EPSRC M.Sc. scholarship in 2001-2002, an EPSRC Doctoral Training Award and an Xyratex Ltd., studentship in 2003-2007, and a CSEL-London Business School scholarship in 2007 to investigate the commercial potential of his research. He was offered a Canon Foundation in Europe fellowship in 2007, and he is currently a Marie Curie Fellow at CERN.

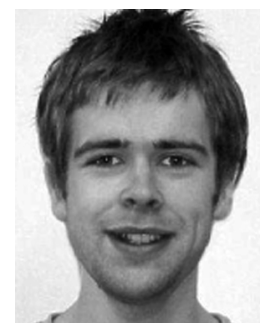

Richard James received the M.Eng. degree in electronic and electrical engineering and the $\mathrm{Ph} . \mathrm{D}$. degree from the University College London (UCL), London, U.K., in 2001 and 2006.

He is currently a Research Fellow with the Liquid Crystal Modeling Group at UCL. His research was supported by an Engineering and Physical Sciences Research Council (EPSRC) scholarship. His current research interests include the numerical modeling of liquid crystal hydrodynamics and defect structures and, more recently, microwave devices with liquid crystal substrates. He is the coauthor of about 20 papers in international journals and conference proceedings.

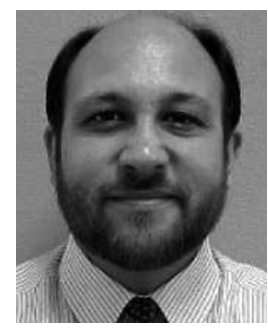

David R. Selviah (M'01) received the B.A. and M.A. degrees in physics and theoretical physics specializing in mathematical modeling from Trinity College, Cambridge University, Cambridge, U.K., in 1980 and 1983 , respectively.

He was an Intern at the Royal Aircraft Establishment, Texas Instruments-Geophysical Service International, and CERN, Geneva, Switzerland. For three years, he was with the Allen Clark Research Center, Plessey (Caswell) Ltd., Northamptonshire, U.K. (now Bookham Technology Ltd.). He then joined the Department of Engineering Science, Oxford University, Oxford, U.K., where he was a Research Fellow for more than three years. In 1987, he joined the Department of Electronic and Electrical Engineering, University College London (UCL), London, U.K., where he is currently a Senior Lecturer. He has more than 150 publications, patents, invited talks, and book chapters $\mathrm{He}$ was engaged in airfield landing lights, aircraft streamlining, experimental particle physics, surface acoustic wave (SAW) RF adaptive correlators, RF filters and radar chirp pulse compression filters, tunable microlenses and optical filters, computer-generated holographic optical elements, dense holographic storage, high sensitivity coherent detection, and LCD backlights. His current research interests include $10 \mathrm{~Gb} / \mathrm{s}$ multimode polymer waveguide optical printed circuit boards with self-aligning multichannel connectors, generalized correlation higher order neural networks and training algorithms for pattern recognition and time series prediction running on field-programmable gate arrays (FPGAs), and real-time image processing and panoramic video. $\mathrm{He}$ has participated in many collaborative projects with industry, and is currently the Technical Leader of the U.K. $£ 1.3$ million IeMRC Optical Printed Circuit Board Flagship Consortium of three universities and eight companies, and also the Technical Leader of the U.K. TSB Fast Track Digital Art Capture Project on high-resolution panoramic imaging of artworks with seven other partner institutions.

Mr. Selviah is a member of the Institute of Physics, the Optical Society of America, and the European Optical Society. He is a member of the Program Committee of the IEEE Annual Workshop on Interconnections Within HighSpeed Digital Systems (HSDS) and is the Cochairman of the IEEE International Symposium on Photonic Packaging. 